trisomy E, de novo balanced translocations (Turnbull, Laurence, and Gregory, 1973), and Klinefelter's syndrome (Walker, Gregson, and Hibbard, 1971) have been found amongst others. However, although the trisomies all tend to be more common in pregnancies of elderly women, those other than Down's syndrome are likely to be found relatively rarely in view of the high early abortion rate in those other trisomies.

K. M. LaURence, P. J. Gregory, and F. SharP

Department of Child Health and Department of Obstetrics and Gynaecology Welsh National School of Medicine, Heath Park, Cardiff

RRFERENCES

Butler, L. J., Reiss, H. E., France, N. E., and Briddon, S. (1973). Antenatal diagnosis of Patau's syndrome (trisomy 13) including a detailed pathological study of the fetus. Fournal of Medical Genetics, 10, 367-370.

Turnbull, A. C., Laurence, K. M., and Gregory, P. J. (1973). Antenatal diagnosis of fetal abnormality with special reference to aminocentesis. Proceedings of the Royal Society of Medicine, 66, 1115-1117.

Walker, S., Gregson, N. M., and Hibbard, B. M. (1971). Nuclear sex and antenatal diagnosis of Klinefelter's syndrome. Lancet, 2 , 430.

\section{A case of partial trisomy 15}

Summary. A girl with mental retardation but few distinctive physical abnormalities is described. Chromosome analysis revealed an extra small acrocentric chromosome with both long and short arms satellited which was identified as a deleted chromosome No. 15. The origin of this chromosome is discussed and the clinical findings compared with those of previously reported cases of partial trisomy 15.

Many previous reports of cases with an extra Glike chromosome associated with a non-mongoloid appearance have failed to establish the origin of the extra chromosome. With the development of chromosome banding techniques identification of such chromosomes has been made possible. There have been three recent reports in which an extra small acrocentric chromosome has been identified by banding as a deleted chromosome No. 15 (Magenis et al, 1972; Parker and Alfi, 1972; Bucher et al, 1973).

We present a further case of partial trisomy 15 in which the extra small acrocentric chromosome is satellited at both ends.

\section{Case report}

The proposita, born in 1967, is the sixth of seven children of Pakistani parents who are first cousins, their mothers being sisters. The six other children all appeared normal. At the time of the birth the father was 38 and the mother 33 years old. Pregnancy and delivery, at 40 weeks, were normal. Birth weight was $3400 \mathrm{~g}$.

The proposita (Fig. 1) presented at 11 months with delayed development and abnormal limb movements which were convulsive in nature. She had several minor seizures daily. All her developmental milestones had been grossly delayed. She had synophrys and strabismus but no other distinct facial characteristics. The facies were reminiscent, but not diagnostic, of the de Lange syndrome and there was no phocomelia and no

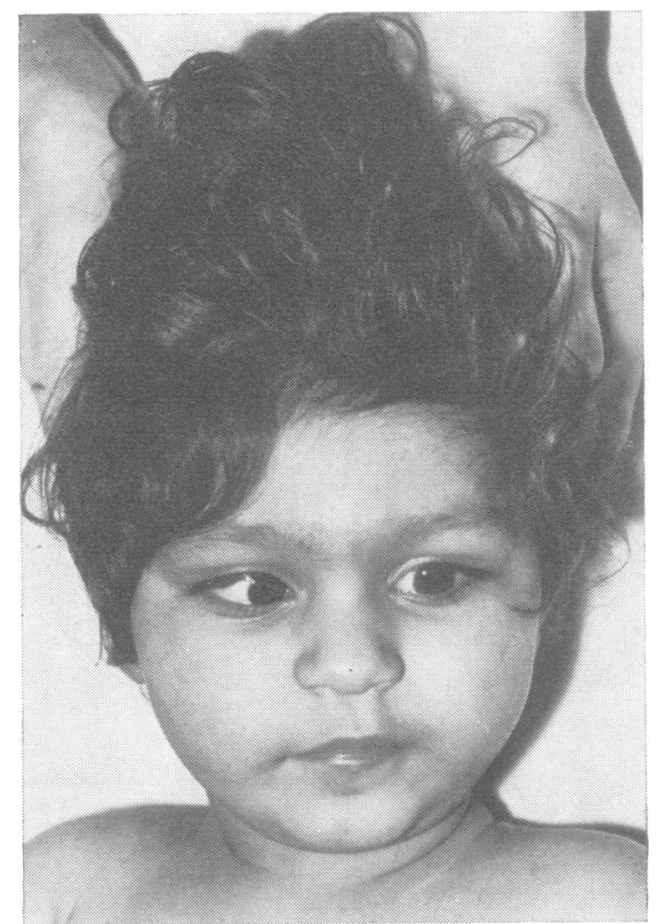

Fig. 1. The proposita. 
other congenital malformations. Head circumference was $42.5 \mathrm{~cm}$ and weight $8250 \mathrm{~g}$. There was gross hypotonia and when pulled to a sitting position there was still head lag. Neurologically there were no other abnormal signs and the fundi were normal.

Laboratory tests revealed normal values for blood counts, electrolytes, plasma urea, blood lead, serum calcium, plasma glucose, and serum and urine aminoacid chromatography. The WR was negative, the CSF normal and the toxoplasma antibodies titre less than 8 . No mercury or metachromatic granules were detected in the urine.

Radiology showed no reduction in size of any of the long bones. The skull was small but there was no intracranial calcification and no premature closures of the sutures. Radiological bone age was about 2 years. An EEG showed spike and slow wave activity which is an epileptic tracing.

\section{Cytogenetic studies}

Chromosome analysis was performed on peripheral blood cultures. Forty-seven chromosomes were counted in 30 orcein-stained metaphase figures from the proposita. The karyotype showed a normal female complement plus an additional small acrocentric chromosome satellited at both ends (Fig. 2). The marker chromosome was identified by trypsin banding (Seabright, 1971) as a No. 15 chromosome with a partial deletion of the long arms and additional unidentified satellite material added on at the breakpoint (Fig. 2). All the other chromosomes showed a normal banding pattern. The break in the No. 15 chromosome appeared to have occurred at q22 and the satellites on the long arms of the extra chromosome seemed to have come from a break in a D- or G-group chromosome just above the centromere. A translocation between the long arms of a No. 15 chromosome and the short arms of an unidentified acrocentric chromosome was postulated.
Orcein-stained karyotypes of the parents and sibs appeared normal. This was confirmed in the parents by trypsin treatment which revealed normal banding patterns.

The association patterns of the acrocentric chromosomes of the proposita were studied using the banded material, the criteria for an association being those of Cohen and Shaw (1967/1968).

\section{Discussion}

The karyotype of the proposita contains the normal female complement plus an extra chromosome identified as a deleted chromosome No. 15 with satellite material on the end of the long arms. The marker chromosome is the product of a translocation between a No. 15 chromosome and another acrocentric chromosome. Since it is not present in either of the parents it must be the result of a new translocation. Two hypotheses were suggested to explain the origin of this chromosome.

1. The translocation between a No. 15 chromosome and another acrocentric chromosome may have occurred in an early cleavage division of the zygote with subsequent non-disjunction of the normal No. 15 chromosome and the monosomic cell line being lost.

2. The marker chromosome may have been present in one of the gametes having arisen from a translocation in meiosis with the resultant quadrivalent undergoing 3:1 segregation.

The karyotype can only be explained by the first hypothesis if the other translocation product had not been formed, leaving an acrocentric chromosome with deleted short arms and an acentric fragment which would be lost in subsequent divisions. A deletion of the short arms of an acrocentric chromo-

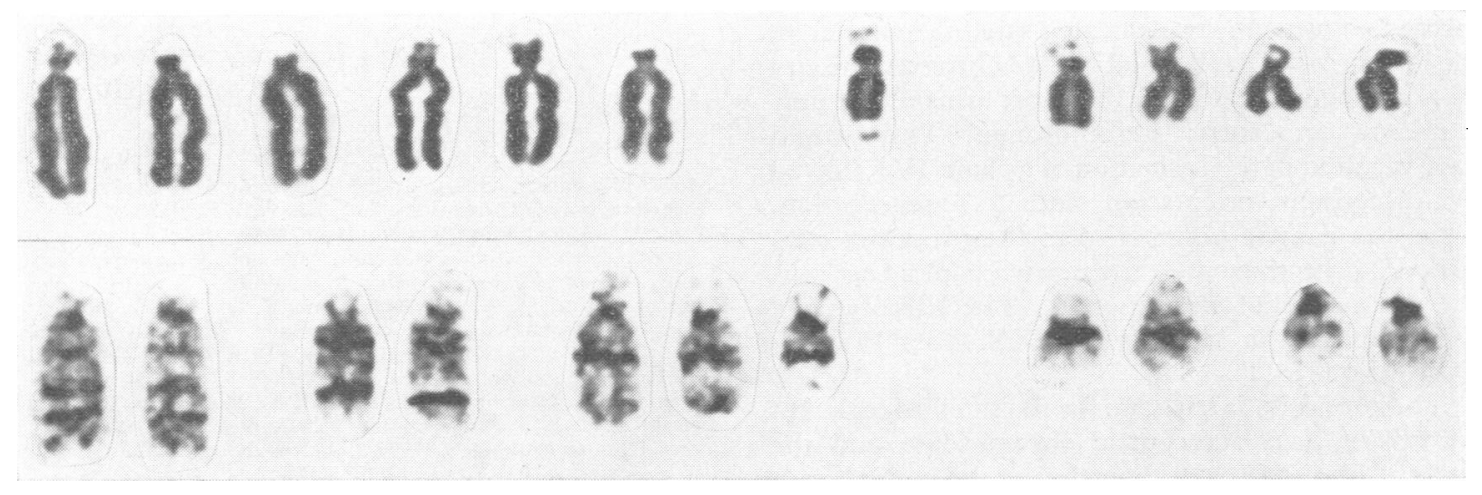

FIG. 2. Unbanded and banded partial karyotypes showing D- and G-group chromosomes and marker chromosome. 


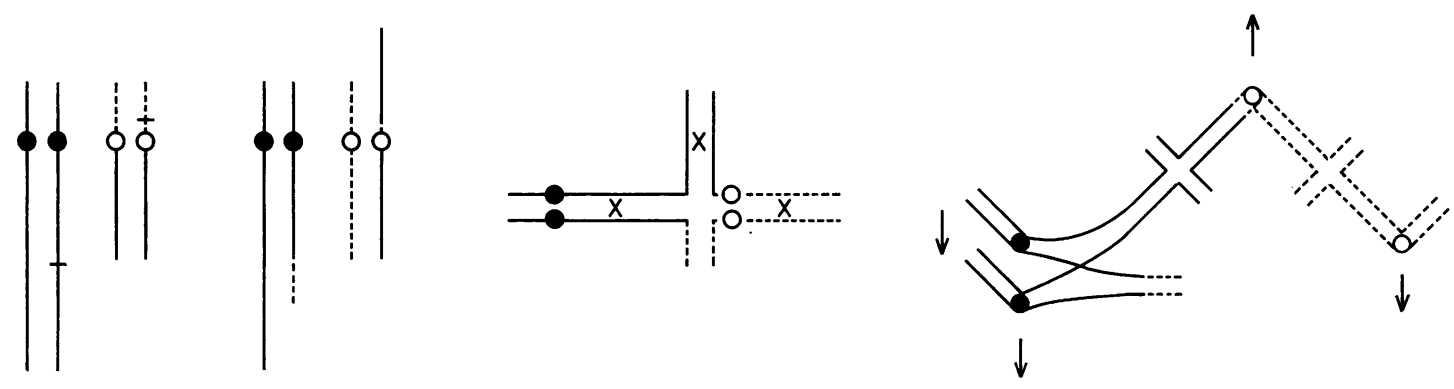

FIG. 3. Suggested segregation of a translocation between a No. 15 chromosome and a G-group chromosome.

some would be difficult to detect using orceinstaining and trypsin-banding techniques but could be detected by its inability to enter into satellite association. Feguson-Smith and Handmaker (1961) found that all five pairs of acrocentric chromosomes are satellited on their short arms and capable of entering into an association. In 30 cells from the proposita both homologues of each pair of acrocentric chromosomes were seen in association in the same cell at least twice and therefore an acrocentric chromosome with deleted short arms cannot be present. Each end of the marker chromosome was capable of entering into an association and in some cells both ends were seen participating in an association at the same time.

Since none of the D- and G-group chromosomes appeared to have deleted short arms the first hypothesis can be discounted and the translocation must have occurred during meiosis I before fertilization. A possible meiotic situation is seen in Fig. 3. This type of translocation, involving acrocentric chromosomes and resulting in an asymmetrical pachytene figure, is likely to undergo 3:1 segregation (Hamerton, 1971). Chiasmata are unlikely to have been formed between the translocated satellite material on the long arms of the deleted No. 15 chromosome and its homologous region on the short arms of the unidentified acrocentric chromosome. This would have resulted in the formation of a chain IV followed by discordant orientation and 3:1 segregation. Thus the gamete giving rise to the proposita contained 24 chromosomes, the normal haploid set plus the extra G-like chromosome. The karyotype of the proposita is therefore $47, \mathrm{XX},+\operatorname{der}(15), \mathrm{t}(15$; $D$ or $G)(q 22 ; p 11)$.

The proposita is trisomic for the satellite region of an unidentified acrocentric chromosome and the short arms and proximal portion of the long arms of chromosome No. 15. Since it has not been shown that trisomy of satellite regions is of any clinical significance our case can be compared directly to other cases of partial trisomy 15. There have been three reports of cases of partial trisomy 15 identified by banding. Two of these (Parker and Alf, 1972; Bucher et al, 1973), in common with our case, showed no abnormal facies apart from strabismus and no congenital abnormalities. They shared with our case mental retardation and a lack of any other distinctive features. However, Magenis et al (1972) described a case identified by banding as partial trisomy 15 with mental retardation and numerous congenital abnormalities. Although no break pointse were identified in these reports, the extra chromosome is similar in size in all cases. However, the lack of distinct clinical features in our case and those of Parker and Alfi (1972) and Bucher et al (1973) is clearly contrary to the findings of Magenis et al (1972).

E. JANET WATSON

The Centre for Human Genetics, 'Langhill', 117 Manchester Road, Sheffield S10 5DN

\section{R. R. GORDON The Children's Hospital, Western Bank, Sheffield S10 2PH}

\section{REFERENCES}

Bucher, W., Parker, C. E., Crandall, B., and Alfi, O. S. (1973). Partial trisomy of chromosome 15. Lancet, 1, 1250.

Cohen, M. M. and Shaw, M. W. (1967/1968). The association of acrocentric chromosomes in 1000 normal human male metaphase cells. Annals of Human Genetics, 31, 129-140.

Ferguson-Smith, M. A. and Handmaker, S. D. (1961). Observations on the satellited human chromosomes. Lancet, 1, 638-640. Hamerton, J. L. (1971). Human Cytogenetics, 1, 269-271. Academic Press, London and New York.

Magenis, R. E., Overton, K. M., Reiss, J. A., Macfarlane, J. P., and Hecht, F. (1972). Partial trisomy 15. Lancet, 2, 1365-1366.

Parker, C. E. and Alfi, O. S. (1972). Partial trisomy of chromosome 15. Lancet, 1, 1073.

Seabright, M. A. (1971). A rapid banding technique for human chromosomes. Lancet, 2, 971-972. 